\title{
Effectiveness of Modified Quadriceps Femoris Muscle Setting Exercise for the Elderly in Early Rehabilitation after Total Knee Arthroplasty
}

\author{
DuJin PARK, MS, PT ${ }^{1)}$, JeOngheE KIM, MS, PT ${ }^{2)}$, HyunOK LEE, PhD, $\mathrm{PT}^{1)}$ \\ 1) Department of Physical Therapy, College of Health Sciences, Catholic University of Pusan:9, Bugok \\ 3-dong, Geumjung-gu, Busan, 609-757 Republic of Korea. \\ TEL:+82 51-510-0570,FAX: +82 51-510-0578,E-mail: holee@cup.ac.kr \\ 2) Busan St. Mary's Medical Center
}

\begin{abstract}
Purpose] This study examined the effects of a conventional quadriceps setting exercise (CQS) and a modified quadriceps setting exercise (MQS) on the strength of the quadriceps femoris, hamstring and gluteus maximus muscles, and the walking ability of elderly persons with osteoarthritis (OA) during their early rehabilitation after total knee arthroplasty (TKA). [Subjects] The subject of this study were 44 elderly women aged 65 or over who were scheduled to undergo TKA on one knee and who voluntarily consented to participate in this experiment after listening to its purpose and method. We divided the subjects into an experimental group $(\mathrm{n}=22)$ who performed MQS and a control group $(n=22)$ who performed CQS. [Methods] We measured subjects' muscle strength and conducted the 6-minute walk test (6MWT) before surgery, 2 weeks after surgery, and 4 weeks after surgery. [Results] Changes in the strength of the hamstring and gluteus maximus muscles were significantly different between the two groups. [Conclusion] MQS may enhance the strength of the hamstring as well as gluteus maximus muscles and is considered an appropriate exercise for the elderly in their early rehabilitation after TKA.
\end{abstract}

Key words: Total knee arthroplasty, Modified quadriceps setting exercise, Muscle strength

(This article was submitted Aug. 3, 2011, and was accepted Aug. 27, 2011)

\section{INTRODUCTION}

Osteoarthritis (OA) is a major cause of physical disability and most frequently occurs in the knee joints. Severe OA restricts elderly persons' routine activities. A common treatment method for severe OA is total knee arthroplasty (TKA). Scientific and clinical evidence recommends TKA for not only alleviating patients' symptoms of and pain from OA, but also for increasing their quality of life $\mathrm{e}^{1,2)}$. Although TKA definitely reduces the pain experienced by elderly persons with knee OA and improves their knee function, it is very rare for their muscle strength and function to recover to normal levels after the operation $^{3,4)}$. Furthermore, sarcopenia, the gradual loss of muscle size associated with aging, is another factor that may result in functional impairment in the elderly).

In this respect, recovery of their muscle strength is crucial, to enhance the functions of the elderly. In particular, the strength of the quadriceps is related with normal activities like climbing stairs; therefore, it is all the more important after $\mathrm{TKA}^{6}$. Atrophy of the quadriceps femoris muscles ranges from $5 \%$ to $20 \%$ within 1 month after TKA compared with the preoperative level, and the strength of voluntary contraction and reduction of cross-sectional area also occur ${ }^{7)}$. In order to prevent amyotrophia in the early stage after surgery, isometric movement of the quadriceps femoris muscles, called quadriceps setting exercise (QS), is widely used for early rehabilitation after TKA or anterior cruciate ligament (ACL) injury. However, Nakajima et al. ${ }^{8)}$ reported that conventional quadriceps setting exercise (CQS) causes shear force to occur at the tibialis anterior, possibly resulting in excessive ACL strain. Also because CQS induces almost no activity in the of hamstring, an antagonist of the quadriceps femoris muscles, CQS places high pressure only on the anterior articular surface and not the total articular surface. Accordingly, they ${ }^{8)}$ devised a modified quadriceps setting exercise (MQS) that contracts the hamstring and gluteus maximus muscles at the same time in order to resolve the lack of activity problem occurring during CQS.

Quadriceps femoris and hamstring muscles provide absorption of impact on tibiofemoral joints and are involved in maintaining functional stability ${ }^{9}$. After TKA, the strength of the hamstring muscles as well as the quadriceps femoris muscles decreases notably ${ }^{6,10)}$; therefore, balanced improvement in the two muscles' strength is necessary. In addition, the hip musculature appears to play a significant role in transferring forces from the lower extremities to the pelvis and spine, acting as one link within the kinetic chain, particularly for all ambulatory activities ${ }^{11)}$. Accordingly, 
this study sampled a population of elderly persons with OA who were scheduled to receive early rehabilitation after TKA and examined the effects of CQS and MQS on the strength of their quadriceps femoris, hamstring, and gluteus maximus muscles and their walking ability.

\section{SUBJECTS AND METHODS}

The subjects of this study were 44 elderly women aged 65 or over who were scheduled to undergo TKA and who voluntarily consented to participate in this experiment after listening to its purpose and method. Eligible subjects included those who could walk alone with or without a cane, had knee flexion contracture of $<10$ degrees, were able to communicate, and had no disease in the other parts of the body except for their knee joint.

We randomly assigned hospitalized subjects who were scheduled to undergo TKA to an experimental group $(n=22)$ or a control group $(n=22)$. We performed conservative physical therapy intervention and MQS for the experimental group and conservative physical therapy intervention and CQS for the control group. In CQS, isometric contraction of the quadriceps femoris was maintained for 10 seconds with the knee joint of the leg that was operated on extended and the ankle dorsiflexed. In MQS, CQS was performed by the leg that was operated on while maintaining the hip and knee joints at 90 degrees flexion, with a 2-kg sandbag placed on the ankle of the opposite leg. According to Nakajima et al. ${ }^{8)}$, application of MQS with a 2 -or $5-\mathrm{kg}$ sandbag placed on the ankle led to a larger increase in muscle activity than application of MQS without a sandbag. Based on their result, we conducted MQS with a 2-kg sandbag because of the age of the subjects. We performed continuous passive motion (CPM) for all subjects while they were in their hospital beds for 1 hour per day at an interval of once every 45 seconds from the day after surgery to the second week after surgery. Then, from the second week to the fourth week, we placed a sandbag weighing about $1 \mathrm{~kg}$ on their ankle, and we administered conservative physical therapy such as resistance training for flexor and extensor muscles of the knee joint and cycling 1 hour per day five times per week. We took precautions to avoid causing pain in the knee joint. From the day after surgery to the fourth week after surgery, we conducted MQS with the experimental group with a 2-kg sandbag placed on subjects' ankles. We performed 10 repetitions of MQS in one set, and we performed three sets of MQS. In order to prevent muscle fatigue, we allowed a 1 minute rest period between sets. The control group received the same conservative physical therapy as the experimental group from the day after surgery to the fourth week after surgery, and we conducted CQS with the same frequencies of exercise and resting time as those of the experimental group.

To measure the strength of the quadriceps femoris, hamstring, and gluteus maximus muscles, we used a handheld dynamometer (Commander ${ }^{\mathrm{TM}}$ Muscle Tester, JTECH Medical, Inc., USA). This instrument is a reliable and valid tool for measuring the muscle strength of elderly persons' hip and knee joints ${ }^{12)}$, and its small size and simplicity make it very clinically useful. The posture used
Table 1. General characteristics of subjects $(\mathrm{N}=44)$

\begin{tabular}{lcc}
\hline \multicolumn{2}{c}{ Experimental Group $(\mathrm{n}=22)$} & Control Group $(\mathrm{n}=22)$ \\
\hline Age $($ years $)$ & $70.3 \pm 3.0$ & $69.1 \pm 3.6$ \\
Height $(\mathrm{cm})$ & $153.2 \pm 4.8$ & $154.6 \pm 6.3$ \\
Weight $(\mathrm{kg})$ & $55.3 \pm 6.7$ & $57.2 \pm 6.8$ \\
BMI $\left(\mathrm{kg} / \mathrm{m}^{2}\right)$ & $23.5 \pm 1.8$ & $24.0 \pm 2.0$ \\
\hline
\end{tabular}

Mean \pm SD; BMI: Body mass index.

for the muscle strength test with the handheld dynamometer followed Reese's method ${ }^{13)}$. The same examiner measured each muscle's strength during maximum isometric contraction maintained for 3 seconds. Measurements were made three times and averaged.

In this study, we conducted a 6-minute walking test $(6 \mathrm{MWT})^{14)}$ to measure the subjects' walking and motor abilities and functional state. The distance of walking in 6 minutes at maximum speed along a $30-\mathrm{m}$ lane marked at 1.5 $\mathrm{m}$ intervals was recorded in meters.

We performed repeated measures analysis of variance in order to examine the effects of CQS and MQS on muscle strength and walking ability of the elderly persons with OA during their early rehabilitation after TKA. We analyzed the data using SPSS 12.0 for Windows and values of $p<0.05$ were considered statistically significant.

\section{RESULTS}

Table 1 shows the general characteristics of the subjects, which did not significantly differ between the two groups. Table 2 shows the changes in the strength of each muscle in the two groups. Changes in the strength of the quadriceps femoris muscles significantly differed in accordance with treatment period. Changes in the strength of the hamstring muscles significantly differed in accordance with treatment period, and they were also significantly different between the two groups. The interaction between treatment period and group was significant. Changes in the strength of the gluteus maximus muscles significantly differed in accordance with treatment period and group, and the interaction between treatment period and group was significant.

Table 3 shows the results of changes in the $6 \mathrm{MWT}$ distance. These changes were significantly different in accordance with treatment period, and there was an interaction between treatment period and group.

\section{DISCUSSION}

A major interest in the rehabilitation process after TKA lies in the strength of the quadriceps femoris muscles, which does not recover well even a few years after surgery ${ }^{10,15)}$. This loss of strength loss results from a combination of muscle atrophy and neuromuscular activation deficits ${ }^{7}$.

A according to Mizner et al., the strength of the quadriceps femoris muscles 1 month after TKA is about $60 \%$ of that before the surgery, despite rehabilitation training within 24 hours of surgery ${ }^{7}$. In this study, the 
Table 2. Changes of muscle strength between the two groups $(\mathrm{N}=44)$

\begin{tabular}{|c|c|c|c|c|c|}
\hline & & & uscle Strength & & \\
\hline & & Baseline (pre) & 2 weeks & 4 weeks & \\
\hline & EG & $19.39 \pm 1.67$ & $12.38 \pm 1.35$ & $15.04 \pm 1.61$ & $\begin{array}{l}\text { Period* } \\
\text { Group }\end{array}$ \\
\hline QD & $\mathrm{CG}$ & $18.85 \pm 1.41$ & $12.50 \pm 1.43$ & $15.07 \pm 1.54$ & Period Group \\
\hline & EG & $13.52 \pm 1.17$ & $11.92 \pm 1.29$ & $12.79 \pm 1.16$ & $\begin{array}{l}\text { Period* } \\
\text { Group* }\end{array}$ \\
\hline HS & CG & $13.37 \pm 1.20$ & $10.60 \pm 1.32$ & $11.71 \pm 1.33$ & Period. Group* \\
\hline & EG & $15.28 \pm 1.17$ & $13.85 \pm 1.31$ & $14.63 \pm 1.12$ & $\begin{array}{l}\text { Period* } \\
\text { Group* }\end{array}$ \\
\hline GM & $\mathrm{CG}$ & $15.44 \pm 1.08$ & $12.67 \pm 0.89$ & $13.69 \pm 1.07$ & Period. Group* \\
\hline
\end{tabular}

EG: Experimental group; CG: Control group; QD: Quadriceps; HS: Hamstring; GM: Gluteus maximus; * Significant difference $(\mathrm{p}<0.05)$.

Table 3. Change of 6-minute walking distance between the two groups ( $\mathrm{N}=44)$

\begin{tabular}{lcccc}
\hline & & \multicolumn{3}{c}{ Walk Distance $(\mathrm{m})$} \\
& Baseline (pre) & 2 weeks & 4 weeks & \\
\hline Experimental group & $302.69 \pm 51.96$ & $272.45 \pm 53.29$ & $292.22 \pm 51.27$ & $\begin{array}{c}\text { Period* } \\
\text { Group }\end{array}$ \\
Control group & $308.36 \pm 50.75$ & $261.69 \pm 50.50$ & $285.45 \pm 49.98$ & Period. Group* \\
\hline
\end{tabular}

* Significant difference $(\mathrm{p}<0.05)$

quadriceps femoris strength of both groups at 1 month after surgery was about $80 \%$ of that before surgery. The experimental group had about $64 \%$ and $78 \%$ of preoperative quadriceps femoris muscle strength in the second and the fourth weeks, respectively, and the control group had about $66 \%$ and $80 \%$ in the same periods. We attribute this result to the differences in the composition and frequency of the early rehabilitation program. In a prior study ${ }^{7}$, light isometric training and active assisted exercises like heel sliders, quadriceps isometrics, hip extensor isometrics, and ankle pumps were performed together for 4 weeks after surgery. Our present study focused on 4 weeks exercise aiming to improve muscle strength using CQS and MQS in addition to basic QS along with general management. QS may increase the activities of muscles such as the vastus medialis obliquus (VMO), vastus medialis (VM) and vastus lateralis (VL) more than diverse straight leg raise (SLR) exercises that can be performed in early training after $\mathrm{TKA}^{16)}$. Thus, QS may lead to an improvement in lateral patellar stability that may result from VM and VMO weakness in the early stage after TKA, because QS minimizes the disuse-related atrophy of $\mathrm{VM}^{17)}$, which occurs first among the component muscles of the quadriceps femoris. Moreover, in this study, we conducted exercise more frequently at five times per week than that of a previous study, three times per week. This may have further helped the recovery of quadriceps femoris muscle strength.

Changes in the strength of the hamstring muscles were significantly different between the two groups. Compared with preoperative levels, subjects' relative strength in the experimental group was $88 \%$ in the second week and $95 \%$ in the fourth week, whereas subjects' strength in the control group was $79 \%$ and $88 \%$ at the same times. This supports the results of a previous study ${ }^{8)}$ that MQS increases the activity of the hamstring muscles more than CQS. Even though changes in the strength of the hamstring muscles were significantly different in accordance with treatment period, their preoperative strength was not different from their strength in the fourth week after operation. Since it has been previously reported that the strength of the hamstring muscles recovers more rapidly than that of the quadriceps femoris muscles ${ }^{4,18)}$, we consider that resilience of the hamstring muscles and the effect of MQS increased the overall strength of the hamstring muscles.

Changes in the strength of the gluteus maximus muscles were also significantly different between the two groups. Compared with preoperative levels, subjects' relative strength in the experimental group was about $91 \%$ in the second week and $96 \%$ in the fourth week, whereas subjects' strength in the control group was about $82 \%$ and $89 \%$ at the same times. This supports the results of a previous study ${ }^{8)}$ that MQS may increase the activities of the gluteus maximus muscles as well as the hamstring muscles. Also, it has been suggested that a dominant pattern of one of two muscles in a force couple, such as the hamstring and gluteus maximus muscles in hip extension, can lead to impairment of the dominant muscle ${ }^{19)}$. In the experimental group, in which we conducted MQS, the relative strength of the hamstring and gluteus maximus muscles was about $95 \%$ and $96 \%$ of preoperative levels, respectively, showing their balanced recovery of strength. Therefore, muscle damage that may occur in hip extension during the stance period may be prevented.

Changes in the 6MWT were not significantly different between the two groups. However, the experimental group showed a faster recovery of 6MWT than the control group at the same times. Compared with preoperative levels, relative 6MWT in the experimental group was $96.5 \%$ in the 
fourth week, whereas relative 6MWT in the control group was $92.6 \%$ at the same time. A possible explanation for this is that MQS assisted improvement in walking ability through strengthening of the hamstring and gluteus maximus. Changes in all muscle strengths and 6MWT distances were significantly different in accordance with treatment period. This suggests that a 4-week general rehabilitation program in the early stage after surgery may not recover muscle strength and walking ability to preoperative levels. In our present study, it was difficult to examine effects of MQS for more than 4 weeks because most of the elderly subjects with TKA were discharged from hospital at 4 weeks after TKA surgery. If the elderly subjects with TKA had continued MQS for 6 to 8 weeks, the results may have been different. Therefore, it will be necessary to study the effects of MQS on muscle strength and walking ability for the elderly with TKA for more than 4 weeks. Furthermore, a recent study ${ }^{20)}$ suggested that rehabilitation programs after TKA should focus on improving the strength of the quadriceps femoris and hamstring muscles. In this regard, MQS may simultaneously enhance the gluteus maximus muscles along with the quadriceps femoris and hamstring muscles; therefore, it may be appropriate for elderly persons' early rehabilitation after TKA. Because MQS may reduce shear force at the tibialis anterior through simultaneous contraction of the quadriceps femoris and hamstring muscles, future research is necessary to demonstrate the effect of MQS as a rehabilitation program after ACL injury.

\section{REFERENCES}

1) Dickstein R, Heffes Y, Shabtai EI, et al.: Total knee arthroplasty in the elderly: patients' self-appraisal 6 and 12 months postoperatively. Gerontology, 1998, 44: 204-210.

2) Jones CA, Voaklander DC, Johnston DW, et al.: Health related quality of life outcomes after total hip and knee arthroplasties in a community based population. J Rheumatol, 2000, 27: 1745-1752.

3) Noble PC, Gordon MJ, Weiss JM, et al.: Does total knee replacement restore normal knee function? Clin Orthop Relat Res, 2005, 431: 157-165.

4) Silva M, Shepherd EF, Jackson WO, et al.: Knee strength after total knee arthroplasty. J Arthroplasty, 2003, 18: 605-611.

5) Volpi E, Nazemi R, Fujita S: Muscle tissue changes with aging. Curr Opin
Clin Nutr Metab Care, 2004, 7: 405-410.

6) Huang $\mathrm{CH}$, Cheng CK, Lee YT, et al.: Muscle strength after successful total knee replacement: a 6- to 13-year followup. Clin Orthop Relat Res, 1996, 328: $147-154$.

7) Mizner RL, Petterson SC, Stevens JE, et al.: Early quadriceps strength loss after total knee arthroplasty. The contributions of muscle atrophy and failure of voluntary muscle activation. J Bone Joint Surg Am, 2005, 87: 1047-1053.

8) Nakajima M, Kawamura K, Takeda I: Electromyographic analysis of a modified maneuver for quadriceps femoris muscle setting with cocontraction of the hamstrings. J Orthop Res, 2003, 21: 559-564.

9) Sharma L, Dunlop DD, Cahue S, et al.: Quadriceps strength and osteoarthritis progression in malaligned and lax knees. Ann Intern Med, 2003, 138: 613-619.

10) Mintken PE, Carpenter KJ, Eckhoff D, et al.: Early neuromuscular electrical stimulation to optimize quadriceps muscle function following total knee arthroplasty: a case report. J Orthop Sports Phys Ther, 2007, 37: 364-371.

11) Lyons K, Perry J, Gronley JK, et al.: Timing and relative intensity of hip extensor and abductor muscle action during level and stair ambulation. An EMG study. Phys Ther, 1983, 63: 1597-1605.

12) Arnold CM, Warkentin KD, Chilibeck PD, et al.: The reliability and validity of handheld dynamometry for the measurement of lower-extremity muscle strength in older adults. J Strength Cond Res, 2010, 24: 815-824.

13) Reese NB: Muscle and sensory testing. New York: Elsevier, 2005, pp 536542 .

14) Bautmans I, Lambert M, Mets T: The six-minute walk test in community dwelling elderly: influence of health status. BMC Geriatr, 2004, 4: 1-9.

15) Meier W, Mizner RL, Marcus RL, et al.: Total knee arthroplasty: muscle impairments, functional limitations, and recommended rehabilitation approaches. J Orthop Sports Phys Ther, 2008, 38: 246-256.

16) Karst GM, Jewett PD: Electromyographic analysis of exercises proposed for differential activation of medial and lateral quadriceps femoris muscle components. Phys Ther, 1993, 73: 286-299.

17) Fox TA: Dysplasia of the quadriceps mechanism: hypoplasia of the vastus medialis muscle as related to the hypermobile patella syndrome. Surg Clin North Am, 1975, 55: 199-226.

18) Berman AT, Bosacco SJ, Israelite C: Evaluation of total knee arthroplasty using isokinetic testing. Clin Orthop Relat Res, 1991, 271: 106-113.

19) Jonkers I, Stewart C, Spaepen A: The complementary role of the plantarflexors, hamstrings and gluteus maximus in the control of stance limb stability during gait. Gait Posture, 2003, 17: 264-272.

20) Stevens-Lapsley JE, Balter JE, Kohrt WM, et al.: Quadriceps and hamstrings muscle dysfunction after total knee arthroplasty. Clin Orthop Relat Res, 2010, 468: 2460-2468. 